\title{
Altern und Arbeit: Förderpolitische Lessons learned
}

\author{
O. Bode und H. Krassen
}

\section{Ausgangssituation}

Engpässe bei Arbeitskräften zu bewältigen und die Beschäftigungsfähigkeit zu erhalten sind unverzichtbare Aufgaben in jedem Unternehmen. Je dynamischer sich die Arbeitswelt weiterentwickelt, umso stärker treten diese Aufgaben in den Mittelpunkt. Die Dynamik am Arbeitsmarkt wird wiederum durch das Zusammenspiel zahlreicher Faktoren vorangetrieben: Digitalisierung, Demografie, Ökologie und gesellschaftliche Umwälzungen sind aktuelle Beispiele. Die Kernprozesse der Arbeit wandeln sich ständig. Hinzu kommt, dass interne und externe Einflussfaktoren auf der Arbeit vielfältiger werden: Sie sind verteilter, wirken asynchron und nicht stationär. Vordefinierte und abgesicherte Lösungsansätze für die Praxis zu entwickeln und anzuwenden, wird hierdurch erschwert. Die Folgen dieser Entwicklungen sind in allen Branchen, Berufen, Tätigkeiten und Qualifikationen spürbar, beispielsweise im Produktionssektor, bei sachbezogenen Dienstleistungen oder in der medizinischen Versorgung und Pflege.

Die demografische Entwicklung und ihre Auswirkungen auf Arbeit und Fachkräftesicherung waren schon immer ein Schlüsselthema der deutschen Wirtschaft und Gesellschaft. 2002 startete das Bundesministerium für Bildung und Forschung (BMBF) eine Demografie-Initiative mit dem Ziel, Management-Instrumente für den betrieblichen Umgang mit dem demografischen Wandel zu entwickeln, zu erproben und zu verbreiten ${ }^{1}$. Die Zielgruppe waren kleine und mittlere Unternehmen (KMU), die unter wissenschaftlicher Begleitung und in Kooperation mit Verbänden, zugängliche Lösungen für die Praxis gemeinsam erarbeitet haben. Bereits damals haben sinkende Geburtenraten, Nachwuchsmangel, Alterung der Belegschaften sowie regionale und betriebliche Spezifika den Handlungsbedarf für die Arbeitsforschung entscheidend geprägt.

Mit der Demografieinitiative und mit ihren Folgeaktivitäten im BMBF konnten seitdem wichtige Kommunikationskanäle für die Streuung von Forschungsergebnissen eröffnet werden. Durch die Überprüfung der Leistungsfähigkeit der entwickelten Instrumente in den Betrieben sowie durch die Einbeziehung von Multiplikatoren, wie Beratende, Personalabteilun- 
gen, Organisationsabteilungen und Sozialpartner, konnte die Breitenwirksamkeit der Forschungsmaßnahmen erhöht und zum Thema Demografie und Arbeit sensibilisiert werden.

Wie vorher skizziert, stellt die Fachkräftesicherung eine der größten Herausforderungen für Deutschland insgesamt dar. Deshalb ist es unverzichtbar, alle in Deutschland vorhandenen Potenziale bestmöglich zu integrieren und prospektiv die Innovationsfähigkeit vor Ort zu fördern ${ }^{2}$ sowie die Herausforderungen hinsichtlich zunehmend alternden und heterogener werdenden Belegschaften zu meistern.

Das Bundesministerium für Bildung und Forschung (BMBF) stellt sich mit der Forschungsprogrammlinie „Zukunft der Arbeit" ${ }^{\text {“3 }}$ im Rahmen des Forschungsdachprogramms des BMBF „Innovationen für die Produktion, Dienstleistung und Arbeit von morgen" ${ }^{4}$ auch heute dieser Aufgabe. Es fördert die Forschung zur Gestaltung neuer Formen der Arbeit, um technische und soziale Innovation zu ermöglichen und die Wettbewerbsfähigkeit des Standorts Deutschland zu sichern. Die Programmlinie „Zukunft der Arbeit" wird mit ESF-Mitteln ${ }^{5}$ kofinanziert. Ihr wichtigstes Anliegen ist es, neue Konzepte der Arbeitsgestaltung und -organisation zwischen Forschung und betrieblichen Partnern mit und für die Praxis zu entwickeln und die Ergebnisse in die Anwendung zu überführen.

Vor dem Hintergrund des demografischen Wandels, einer Beschleunigung des technologischen Wandels sowie der Globalisierung soll die Innovationsfähigkeit der Unternehmen aus der Verknüpfung von Personal-, Organisations- und Kompetenzentwicklung gestärkt werden. Damit hilft die Programmlinie, zukunftsfähige und zugleich sozialverträgliche Arbeitsplätze zu schaffen und die Innovationsfähigkeit im Land zu stärken.

Die Folgen der Veränderungen der Altersstruktur auf die Arbeitswelt und die neuen Anforderungen an die alters- und alternsgerechte Arbeitsgestaltung sind wichtige Aspekte in der Programmlinie „Zukunft der Arbeit“. Sie werden aus vielfältigen Perspektiven betrachtet. Es geht um Fragen wie präventive und gesundheitsförderliche Arbeitsgestaltung, Schnittstellen für die Mensch-Maschine-Interaktion, neue Arbeitsformen im Datennetz und Kompetenzentwicklung im Arbeitsprozess.

Insbesondere der Aufbau von Kompetenzen ist zur Lösung komplexer Probleme, zur Ausübung neuer Formen von Ermessensspielräumen oder zur beteiligungsorientierten Kollaboration von Mensch(en) mit technischen Systemen von essentieller Bedeutung. Ziel ist es, lebenslanges Lernen als unverzichtbare Grundlage für den Erhalt der Beschäftigungsfähigkeit zu etablieren. 


\section{Impulse und Erkenntnisse: Was haben wir gelernt?}

Innovative Kompetenzmodelle können dabei helfen, neue Lösungsstrategien zu identifizieren, um die innerbetrieblichen und im Unternehmensnetzwerk enthaltenen Potenziale zu nutzen. Vor allem für kleine und mittlere Unternehmen (KMU) ist es wichtig, Kompetenzmanagement und dessen Ansätze strategisch zu verankern und die Instrumente am jeweils gegebenen Entwicklungsstand bzw. Reifegrad von Beschäftigten und Unternehmensstrukturen selbst anzusetzen.

Im Fokus des Förderschwerpunkts „Betriebliches Kompetenzmanagement im demografischen Wandel" 6 des BMBF haben 31 Verbünde zwischen 2014 und 2018 die oben genannten Fragestellungen bearbeitet und prototypische Lösungsansätze in realen Anwendungsszenarien erprobt. Nachfolgend wird ein Auszug aus projektbezogenen Thesen und Erkenntnissen dargestellt, die dazu beitragen können, eine innovationsförderliche Unternehmenskultur im demografischen Wandel zu schaffen.

- Beschäftigte müssen sich permanent weiterbilden. Der Erwerb neuer Kenntnisse, Fertigkeiten und Kompetenzen wird für die Beschäftigten immer wichtiger. Traditionelle Weiterbildungskonzepte greifen hier zu kurz. In der Arbeitswelt von morgen spielen vor allem eigenständiges Arbeiten, lebenslanges Lernen und eine im Arbeitsprozess stattfindende aktive Kompetenzentwicklung eine große Rolle. Betriebliches Kompetenzmanagement heißt daher: Weg von traditioneller Aus- und Weiterbildung hin zur Entwicklung von Kompetenzen mit innovativen Lernformen.

- Arbeit muss für alle Altersgruppen lern- und gesundheitsförderlich gestaltet werden: Sie muss hohen körperlichen Fehlbeanspruchungen genauso entgegenwirken wie auch einer permanenten Überforderung infolge mangelnder Kompetenzen. Die Beschäftigungsfähigkeit ist dabei sowohl für Berufsgruppen als auch altersspezifisch differenziert zu betrachten.

- Die Möglichkeiten digitaler Technologien, moderner Lernformate und nachhaltiger Kompetenzentwicklungsprozesse sind arbeitsintegriert zu erschließen. Einen guten Ansatz dazu können mediengestützte Arbeitsund Lernmodule darstellen, beispielsweise die Integration berufspädagogischer Inhalte an hochtechnisierten Anlagen in den realen Produktionsprozess. Dies setzt die Bereitstellung von Know-how und Ressourcen sowie den Aufbau einer notwendigen Infrastruktur im Arbeitsumfeld voraus. 
- Die lebensphasenspezifischen Ansprüche der Beschäftigten an Arbeit und Kompetenzentwicklung nehmen zu. Beschäftigte in allen Altersgruppen können durch die Vermittlung fachlicher, methodischer und sozialer Kompetenzen nachweislich dauerhaft beschäftigungsfähig gehalten werden. Dabei müssen gruppen- und altersspezifisch geeignete Rahmenbedingungen und Lehr-Lernformate geschaffen bzw. eingesetzt werden. Dabei ist die Zusammenarbeit älterer und jüngerer sowie technikaffiner und technikaverser Mitarbeiterinnen und Mitarbeiter von Vorteil.

- Merkmale der demografischen Entwicklung treten verstärkt auch in der Sozialwirtschaft, insbesondere der Kinder- und Jugendhilfe, auf. Ältere Beschäftigte in der Kinder- und Jugendhilfe stehen vor der Frage, ob und wie sie Beruf und Lebensalter in Einklang bringen können. Es gilt, erfahrene Fachkräfte leistungsfähig in diesem anspruchsvollen Berufsleben zu halten und die Qualität der Kinder- und Jugendhilfe mit maßgeschneiderten Instrumenten der Personal- und Organisationsentwicklung auf allen Organisationsebenen bei den Trägern zu gewährleisten. Dabei können sich bisher kleinteilige Organisationen zu Elementen einer Leistungskette entwickeln, ihre Handlungsfähigkeit erweitern und zusätzliche Gestaltungsspielräume erhalten, insbesondere auch in strukturschwachen Regionen.

- Pflegekräfte sind die wichtigste betriebliche Ressource für Altenheime, Pflegedienste und Krankenhäuser. Ausgerechnet diese Ressource kann derzeit nicht ausreichend gesichert werden: Kranken- und Altenpfleger/ innen scheiden überdurchschnittlich früh aus dem Erwerbsleben aus, unterbrechen ihre Erwerbstätigkeit so häufig wie kaum eine andere Berufsgruppe oder arbeiten oft nur in geringem Stundenumfang. Ursächlich hierfür sind Überforderung, innere Kündigung bis hin zum Burnout. Offensichtlich benötigen Kranken- und Altenpfleger/innen adäquate Kompetenzen zur Bewältigung diverser anspruchsvoller Anforderungen in einem hochverdichteten komplizierten Arbeitsumfeld, wie Kompetenzen zur effektiven Steuerung von Veränderungsprozessen sowie zum Entscheidungs- und Konfliktmanagement. Deshalb sind hier neue wirksame Kompetenzen schon bei der beruflichen Bildung zu fördern und in der Praxis umzusetzen.

- Neue, selbstständige Formen der Arbeit wie virtuelle, Tele-, Mobiloder Projektarbeit verbreiten sich immer weiter und die Zahl (Solo-)Selbstständiger nimmt zu. Diese individualisierten Arbeitsbedingungen betreffen vor allem hochqualifizierte Fachkräfte, insbesondere Frauen und ältere Beschäftigte, deren Erwerbsbiografien flexibler werden. Die Arbeitenden gestalten zu immer größeren Anteilen eigenver- 
antwortlich ihre Arbeitsbedingungen mit. Um die damit verbundenen Spielräume effektiv und sinnvoll nutzen zu können, benötigen sie spezifische Angebote der Gestaltungskompetenz.

- Industrielle Dienstleistungen sind für den Maschinen- und Anlagenbau zu einem zentralen Geschäftsfeld und entscheidenden Wettbewerbsfaktor geworden. Mit dem Ausbau und der Professionalisierung von industriellen Dienstleistungen entsteht ein Bedarf nach speziellen Kompetenzen, die neben fachlichen verstärkt methodische und soziale Kompetenzen einschließen. Diese Entwicklung stellt Maschinen- und Anlagenbauer vor die Herausforderung, den bisherigen Fokus auf neue Kompetenzfelder auszuweiten. Gleichzeitig stehen Maschinen- und Anlagenbauer zunehmend vor der Herausforderung, die Beschäftigungsfähigkeit älterer Kompetenzträger zu sichern und neue Karrierewege innerhalb des Servicebereichs zu schaffen. Unternehmen sind beispielsweise durch den steigenden Automatisierungsgrad und komplexere Technologien herausgefordert, wichtiges betriebliches Erfahrungswissen für Produktionsabläufe proaktiv zu erhalten und zu erneuern, um Unternehmenserfolge zu sichern.

- Moderne Unternehmen sind auf kompetente Mitarbeiter und Mitarbeiterinnen angewiesen, die sich durch eine stark ausgeprägte psychische Widerstandsfähigkeit (Resilienz) im Umgang mit sich ständig verändernden Anforderungen auszeichnen. Instrumente, die die Resilienz von Beschäftigten aller Altersstufen in Unternehmen ausbauen, sind zu implementieren, zum Beispiel Diagnoseinstrumente, Resilienz-Zirkel und altersangemessenes (E-)Coaching.

- Eine Neuerung stellt der arbeitsprozessintegrierte Kompetenzerwerb durch prozessbegleitende gemeinsame Produktentwicklung jüngerer und älterer Beschäftigter dar. Es wird systematisch an das Erfahrungswissen von Beschäftigten angeknüpft. Dazu werden neuartige flexible betriebliche Lernformen eingesetzt sowie Produkt-, Lern- und Teamentwicklungsworkshops durchgeführt. Im Mittelpunkt stehen die Integration in den Arbeitsprozess, neue betriebliche Erfahrungsräume und heterogene Lernkonstellationen („mit einem Anderen lernen“) durch die gemeinsame exemplarische Arbeit an einem innovativen Produkt. Beschäftigte tätigen gemeinsame Innovationsarbeit entlang des gesamten Produkterstellungsprozesses. Die Unternehmen müssen hier eine aktive Rolle spielen und Lernpartnerschaften sowie Kompetenzen zur Selbstreflektion fördern.

- Eine Möglichkeit für die nachhaltige Ausschöpfung wertvoller Kompetenzen besonders älterer, berufserfahrener Beschäftigter bietet sich in Unternehmen durch „zweite Karrieren“ und berufsbegleitende Ent- 
wicklung der dafür fehlenden Kompetenzen. Eine Schlüsselfunktion nimmt die systematische Dokumentation und Anerkennung der im Arbeitsleben erworbenen Kompetenzen ein.

- Im demografischen Wandel müssen besonders auch die Kompetenzen der unteren Hierarchieebenen (Shop Floor) in der Produktion aktiviert und gefördert werden. Letztlich macht das facettenreiche (Erfahrungs-)Wissen an der Basis den Erfahrungsschatz eines Unternehmens aus. Möglichst breit einzusetzen ist ein Kompetenzmanagementkonzept, mit dem im Sinne eines „Shop Floor Managements“ systematisch in Werkstätten und altersgemischten Produktionsteams, das Wissen und die Erfahrung von vielen erschlossen wird. Das Konzept verbindet Technikdidaktik, Arbeitswissenschaften und Produktionsmanagement.

- In strukturell schrumpfenden Regionen der Bundesrepublik wird Facharbeit zu einem „regionalen Kollektivgut“, das es zu erhalten gilt.

- Die Personalarbeit im Unternehmen muss systematisch entwickelt und gegebenenfalls „professionalisiert“ werden. Personalplanung, alter(n)sgerechte Arbeitsgestaltung und Qualifizierung, Know-how Transfer, Kompetenzentwicklung sollen Veränderungsprozesse proaktiv und strategisch unterstützen.

\section{Ausblick: Neue Formen der Wertschöpfung und Arbeit möglich machen}

Die Arbeit der Zukunft zu gestalten, kann sich nicht alleine auf den Umgang mit neuen Technologien beziehen. Sie muss das gesamte sozio-technische System betrachten und die Frage danach stellen, wie das soziale Handeln und ein neues Wechselverhältnis von Technik und Gesellschaft durch Veränderungen konfiguriert wird. Innerbetrieblich bewährt haben sich partizipative und auf Vertrauen basierende Gestaltungsprozesse, die alle beteiligten Akteursgruppen einbeziehen. Überbetrieblich werden ähnliche Ansätze entwickelt und verfolgt, die Kunden und Zivilgesellschaft integrieren - durchaus entlang bestehender und sich neu formender Wertschöpfungsketten bzw. Wertschöpfungsnetzwerke.

Qualifizierung und Kompetenzentwicklung bleiben dabei auch weiterhin ein Schlüsselfaktor, um die wirtschaftlichen Potenziale der Digitalisierung zu heben und faire Zugangschancen zu den Arbeitsmärkten der $\mathrm{Zu}$ kunft zu eröffnen. Für Maßnahmen zur Kompetenzentwicklung bietet es sich an - wie vorher beschrieben - sie demografisch sensibel aufzubauen. Dabei geht der Begriff „Demografie“ weit über die Frage nach dem Alter hinaus. Er berücksichtigt beispielsweise auch sprachliche, kulturelle, berufsbiografische, gleichstellungsrelevante Aspekte etc. 
Eine immer komplexer werdende Arbeitswelt erfordert - sowohl von den Unternehmen wie auch von ihren Beschäftigten - eine breite und dynamische Interpretation des notwendigen Kompetenzerwerbs:

- Fach- und Methodenkompetenzen (z. B. Umgang mit mobilen Endgeräten zur Steuerung von Produktionsanlagen, EDV-Kenntnisse, Sprachkenntnisse),

- Sozialkompetenzen (z. B. Anlernen von KollegInnen, Teamfähigkeit, Kommunikationsfähigkeit, interkulturelle Kompetenz)

- Selbstkompetenzen (z. B. Bereitschaft zu lebenslangem Lernen, Kompetenz zum selbstgesteuerten Lernen - eigenständiges Festlegen von Lernzielen, eigenständige Auswabl geeigneter Lernstrategien, eigenständige Kontrolle des Lernerfolgs).

Es steht außer Frage, dass neue technologische Entwicklungen wie die Instrumente der Künstlichen Intelligenz (KI) das Potenzial haben, Wissenschaft, Wirtschaft und Gesellschaft bei der Bewältigung der Herausforderungen im Kontext des digitalen und demografischen Wandels zu unterstützen. Bei der Einführung von KI in die Arbeitswelt ist es wichtig, Chancen und Risiken für das jeweilige Unternehmen und seine Beschäftigten strukturiert, spezifisch und zielgerichtet zu berücksichtigen. Die Forschung in diesem Feld muss Möglichkeiten eröffnen, die als gute Beispiele von den Unternehmen als Prototypen für eigene Entwicklungen genutzt werden können. Einheitsrezepte helfen in der Vielfalt nicht weiter.

Hinter dieser Aussage steht die Überzeugung: Der Mensch wird auch weiterhin die Veränderungsprozesse gestalten und letztlich entscheiden. Gestalten und Entscheiden sind Prozesse, die Alternativlosigkeit nicht kennen. Transparenz, Partizipation und Kommunikation werden auch für zukünftige Arbeitsbeziehungen entscheidende Faktoren sein. Sie schaffen dabei von selbst weitere „Diversität“ und „Pluralität“. Für die Arbeitsforschung werden sie Handlungs- und Anwendungsfelder sein - das gilt auch dann, wenn auch KI die Entscheidungsfindung unterstützen kann.

\section{Referenzen}

1 Schemme, D. Altersgerechtes und generationenübergreifendes Lernen - eine Strategie für Unternehmen, die Personal- und Organisationsentwicklung kombinieren. BWP 2/2003. BIBB. 2003.

2 Jedes Alter zählt. Eine demografiepolitische Bilanz der Bundesregierung. BMI. 2017.

3 Zukunft der Arbeit. Innovationen für die Arbeit von morgen. BMBF. 2016. 
4 Innovationen für die Produktion, Dienstleistung und Arbeit von morgen. BMBF. 2014.

5 www.esf.de.

6 https://www.produktion-dienstleistung-arbeit.de/de/betriebliches-kompetenzman agement-im-demografischen-wandel-1979.html. 